\title{
Editorial
}

\section{A tsunami of solidarity for Japan}

On 11 March 2011 a magnitude 9.0 earthquake struck Japan, near the east coast of Honshu, $129 \mathrm{~km}$ east of Sendai. The earthquake generated a devastating tsunami that swept over cities and farmland along the northern part of Japan. The result has been an immeasurable loss of life and damage to critical infrastructure.

Once again we are humbly reminded that many tragedies around the world often relate to the dynamic nature of planet Earth.

As Earth Scientists, we all well appreciate the need to better understand the causes of natural hazards. Our collective efforts through geoscience education, practical field research and innovative studies have helped to minimize the risks of natural hazards, reduce human vulnerability and enhance the safety of the global society. Moreover, we know that in order to confront natural disasters not only the geosciences but numerous other disciplines are needed to respond to and mitigate such events.

With a long historical record of earthquakes and tsunamis Japan has developed the most stringent safeguards to ensure that buildings and infrastructure are built to be resilient and capable of surviving this type of disaster. Japan has also developed and maintained the organizational capacity and equipment needed for prevention, early warning and rapid emergency response to natural hazards.

This earthquake and its aftermaths, however, show that there is a limit beyond which most efforts are neutralized. Thus, even a society like the Japanese, well prepared for this type of event, may be subject to tremendous losses when the effects of the dynamics of planet Earth reach unusual magnitudes in a given context.

These circumstances surely will not change in the immediate future. On the contrary, as it has already been pointed out by some analysts, not only we will see more natural disasters, but also our human population and built-up settlements could be increasingly vulnerable to them. This vulnerability results from a continuous increase in population, the presence of new and larger human settlements in areas prone to natural hazards, and the development of technologies needed to maintain that population and the standards it requires even at the cost of some actual or potential negative effects on our environment.

What is basically needed then to cope with this type of inevitable events is the ethos that the Japanese have learned since childhood and are showing today as an example for the rest of the World - an ethos in which resilience, perseverance, patience and solidarity are some of the most outstanding features.

On behalf of the many geoscientists represented by the IUGS, we send our heartfelt condolences and sympathies to the many people affected by this recent catastrophe. The Japanese people are not alone; a wave of solidarity is reaching them throughout the globe.

Alberto C. Riccardi President, IUGS 